Research

\title{
A Perspective on the Evolution of e-Dialogues Concerning Interdisciplinary Research on Sustainable Development in Canada
}

\author{
$\underline{\text { Ann Dale }}^{1}$
}

\begin{abstract}
Sustainable development research is inherently interdisciplinary; it requires the conscious search for unifying concepts that foster and reinforce understanding across disciplines. In addition, the number of sectors and actors involved in potential solutions requires a multistakeholder approach to decision making. The challenge of sustainable development research increasingly presents itself as a problem-solving activity. It involves producing useful knowledge through applied research. It is normative and not valuefree. It involves complex issues of polity and culture. Thus, sustainable development research needs novel methods for research, for bringing together expertise that crosses disciplines and sectors, and for informing policy development. It also requires an unprecedented level of integration between the natural and social sciences. This paper describes how the lessons learned from a multistakeholder roundtable have been applied to the development of deliberatively designed, transdisciplinary, electronic spaces for synchronous dialogue on sustainable development and other critical public policy issues. This approach has now evolved into a novel research data collection method for students.
\end{abstract}

Key Words: collaborative inquiry; e-dialogue; interdisciplinary research; multistakeholder process; research methods; sustainable development

\section{INTRODUCTION}

Internet communications technologies (ICTs) have evolved into many diverse forms, including listservs, online conferences, asynchronous discussion groups, young scholar dialogues (Peterson et al. 1997), chat rooms, and blogs. As recently as 1996, however, these methods of communication were usually text-based and asynchronous (Wellman et al. 1996). Some synchronous applications are now emerging, most notably those of the Worldwatch Resources Institute and the e-dialogues dominated by Royal Roads University. This paper explores how the Internet offers a rich medium for both the interdisciplinary research and the substantive dialogue that are crucial to people studying sustainable development.

Interdisciplinary dialogue is critical to sustainable development research because the problems posed in this field cannot be solved by any one sector or discipline. The solutions are inherently interdisciplinary and transdisciplinary. They are normative and value-laden. The challenge, therefore, does not necessarily concern science or management. Instead, the challenge is about dealing with people and their diverse cultures, interests, visions, priorities, and needs (Norgaard 1994).

In some ways, Internet communications mimic ecological systems: they have self-organizing properties, they can bring diverse groups of people together in one space, and their anarchistic characteristics lead to evolutionary change. ICTs, however, have one important difference: they operate independent of place. This property contributes to their interdisciplinary nature by efficiently bringing together diverse intellectual capital in the same space and time to participate in dialogue that often has emergent properties. 
Several lessons have been learned from the multistakeholder processes and asynchronous discussions that have used these synchronous and substantive online dialogues to advance sustainable development research.

\section{MULTISTAKEHOLDER PROCESSES}

In 1988, the Canadian government created the National Round Table on the Environment and the Economy (NRTEE) with a mandate to bring the best minds in the country together around the dissemination and implementation of sustainable development in Canada. Instead of following the traditional institutional model of bringing together individuals or sectors that share common interests or goals, the roundtables were multipartite. Members represented diverse experiences, perspectives, insights, values, and beliefs. NRTEE was the first body in the history of Canadian public administration to deliberately bring together groups from conflicting sectors. The roundtable was based on the belief that a team always outperforms the individual and that, for a group to be smart, it should be autonomous, decentralized, and diverse (Shermer 2004).

The roundtable was a microcosm of society itself, with membership drawn from government, business, environment, and other senior levels of public policy making. Any issue identified by NRTEE as an initiative had to be strategic rather than operational, multipartite and cross-disciplinary, interjurisdictional or interdepartmental, long-term, focused on means rather than ends, and national or international in scope. This unique structure was in place until 1992 when the federal government quietly withdrew its ministers from sitting directly at the table. Since then, NRTEE has continued as a more traditional consultative model.

Although much of the dialogue from this time remains confidential and debate continues over the significance of many of NRTEE's achievements, the roundtable's biggest contribution was the creation of novel networks of civic engagement around the basic concept of sustainable development and the dissemination of this concept by its members in key sectors of Canada. The roundtable's lasting legacy may simply be the creation of an educated elite, or what is sometimes called action learning (Schon 1983, Morgan 1986, Michael 1993) around sustainable development principles and later practices. NRTEE's leadership also led to the creation of more than 1000 municipal-level roundtables, many of which are still in place today.

Roberts and Bradley (1991) have developed the notion of "transmutational purpose" to describe what they believe to be an essential property of collaboration. Transmutational purpose is the idea that parties that work together are involved in refashioning a set of raw materials (objects, ideas, or social relations) into a developed product. NRTEE was also responsible for the creation of the Canadian Consortium for Sustainable Development Research (CCSDR) in 1990. The consortium includes the academic directors of all the major research centers involved in sustainable development research as well as the heads of major teaching and research programs in environmental sciences across the country. The CCSDR continues its work, and it has advanced interdisciplinary thinking and collaboration in sustainable development throughout the Canadian academy.

As one of two civil servants involved in the creation of NRTEE, I observed this experiment in setting up a multistakeholder roundtable firsthand. I noted the value of getting diverse groups of people with different perspectives together around the same table. Perspective-taking appears to be very important in the search for collaborative solutions, and learning to communicate across sectors and disciplines is vital for revealing values and underlying paradigms.

The NRTEE experiment also showed that roundtable members need to pay particular attention to issues of trust, to building social capital, and to different needs with respect to information and detail. For example, corporate leaders on the roundtable often expressed frustration over the amount of information they needed to digest, especially when it concerned the process. Environmental leaders, on the other hand, often suspected that information was being withheld unless they were given very detailed documents.

\section{ELECTRONIC COLLABORATIVE INQUIRY}

From September 1995 to August 1997, I developed a doctoral research project exploring some of the lessons that could be learned from NRTEE. Among 
these were the importance of diversity within collaborative teams; the need for participation by key sectors critical to the implementation and dissemination of team findings; the importance of expertise, particularly interdisciplinary skill, at the table; and the need for expert moderation. One of the weaknesses within the NRTEE membership was a lack of research and scientific expertise. Thus, my research also examined the interface of science and public policy and the ability of these two disciplines to engage in meaningful dialogue with other sectors of society.

In September 1996, I created an online collaborative inquiry involving 20 co-researchers drawn from the policy community. The inquiry was built upon the notion of subjecting a set of ideas to interdisciplinary and intersectoral dialogue. The concept of co-researchers was drawn from "participatory action research" (Rowan 1981a) in which no discrimination is made between subject and object and everyone collaborates in the research process.

The inquiry included academics and activists but not business representives. Because the purpose of the research was to develop a framework for sustainable development governance, collaboration was restricted to those who supported the basic concept. Expertise, perspectives, and values nevertheless differed significantly among the coresearchers. Co-researchers were drawn from public policy, academia, and non-governmental organizations. The selection of co-researchers from these three sectors was deliberate and identified key or emerging leaders who would be committed to the framework development and who would work as advocates for change in each of their sectors. I also believed an academic balance between the natural and social sciences was critical to sustainable development research.

Collaborative inquiry was chosen as a research methodology because it is grounded in dialectical thinking, which works to reveal critical contradictions and paradoxes. The concept of sustainable development is an integration of two terms not previously linked and contains many inherent paradoxes. Not the least among these is the notion of economic growth and progress in a world constrained by natural limits. Many argue that the implementation of sustainable development principles has been impeded by the growth-vs.-nogrowth debate since the 1970s. Dialectical theories actively seek contradictions and paradoxes within situations and use these to expose what is happening and what is likely to happen. They do this at three levels, demonstrating the interdependence of opposites, the interpenetration of opposites, and the unity of opposites (Rowan 1981b). Revealing these opposites was essential to my research.

Electronic collaborative inquiry was chosen as a research tool because it satisfied several personal and professional criteria. First, it is an extremely cost-effective method for bringing many people together. For example, NRTEE had a budget of Can. $\$ 2.5$ million and a staff of 14 to conduct its face-toface meetings and day-to-day operations, a sum to which few organizations and groups have access. Second, electronic collaborative inquiry improves the interdisciplinary nature of the research by eliminating the constraints of time and place, reducing transportation and transaction costs, allowing participation from across the country, and providing an opportunity for diverse geographical perspectives. Third, it eliminates the costs of transcribing because an electronic record is immediately available. Fourth, it is more democratic and objective because it allows for voice to be recorded directly, removing the filter of the researcher from those being researched. Fifth, it addresses some aspects of equity by considering factors of inclusion such as age, regional representation, gender, and representation from different sectors, except the business and labour communities. Despite this, however, the research group in our case included only elite, white, middleclass expertise and experience.

After reviewing several online forums, I selected a simple e-mail method, and a list-serv was created. I opened the dialogue with a series of iterative, openended questions. This method was also used to test whether ICTs could facilitate discussion by providing a "neutral, safe place" in which the absence of traditional physical cues may or may not allow for different ways for voices to emerge. For example, people who are sensitive to dominance patterns may find Internet communication a safer vehicle for expression. Although other dominance patterns may occur on the Internet, they are still nascent and emerging. To optimize the emergent and spontaneous properties of Internet communication for dynamically interactive dialogue, e-mail was chosen because it did not involve going to another site and because it was directly within the sphere of day-to-day activity for the co-researchers who 
consistently and regularly use e-mail correspondence.

\section{LESSONS LEARNED}

At least five significant barriers to effective dialogue emerged in my study: literacy, language, trust, intersectoral communication difficulties, and disciplinary structure.

Literacy was a surprising factor affecting three of my co-researchers. These participants had identified themselves and had asked to be part of the dialogue. They were, however, subsequently inactive throughout the two-year dialogue. In one case, the barrier might have been an age variable that was reflected in a limited ability to type. Typing ability is a major impediment to interacting in an information-rich Internet dialogue. In the other two cases, both individuals were very verbally articulate but lacked written literacy. Academics attach inordinate importance to the written word. In my study, this concern appeared to cause some to pull back from spontaneous and emergent thought, despite my exhortations to the group to be informal and to allow the spontaneity of the medium to emerge. This was a major "sticking point" or cause for silences and reflected a loss of the immediacy of the medium. This immediacy is critically important and compensates for the spontaneity, synergy, and trust that develops during traditional face-to-face interactions.

Language was another major barrier to participation, particularly among the three sectors. Many participants from the field of public policy found the level and tone of the debate too academic. Co-researchers from nongovernmental organizations were intimidated by jargon. This caused one participant from this sector to withdraw. The word "sector" itself provides an example of the importance of language, suggesting hard and demarcated divisions and discrepancies between groups. Using prevailing language that everyone understands is an easy conceptual trap. The concept of vertical stovepipes is useful for describing the boundary setting within institutions that is common to human organizations (Dale 2001). These stovepipes make cross-sectoral discourse problematic. One of the co-researchers in the group often made the point that the academic sector needs to simplify its language if it is committed to the wide dissemination of research. The ability to take complex concepts and communicate them in clear and simple language proved to be an impermeable barrier to substantive dialogue among the three sectors.

Expert moderation was the key to sustaining meaningful dialogue. My online moderation techniques varied from direct active engagement to letting the silences speak. These were sometimes deafening. Before intervening, I often asked myself whether the silences indicated moments of reflection by my co-researchers or whether they were the result of a critical lull, a loss of momentum in the conversation. Thus, a sense of timing and pace was critical for moderation. The absence of physical cues is both a strength and a weakness of Internet dialogue, but it also requires that online moderation use a different set of skills for "sensing" when to facilitate and when to moderate. Knowing when to prompt the group and when to refrain was critical to building dialogue and commitment to the research process. I used a variety of communication styles to facilitate the dialogue, such as alternating between professional and personal messages to alleviate silences. In the latter stages of the dialogue, I used "personal contextualization" and the interjection of my own half-formed ideas to facilitate greater spontaneity and brainstorming and to build online trust.

Most importantly for the research, the electronic medium allowed for continuous cycles of inward and outward contemplation, or analysis and reflection, by the co-researchers. It also allowed for alternating spirals of strategic questioning, critical reflection, and action inquiry through the electronic dialogue. This was followed by information consolidation and further rounds of critical reflection, strategic questioning, and action inquiry through the external peer review process. It provided a space for sustained and reflective dialogue, and it kept a history of these to which people could easily refer.

This research examined both the research outcome, reflected in the eventual framework, and the process of a long-term substantive dialogue using Internet communications technologies. It demonstrated the capacity of the Internet for substantive and sustained dialogue. Two additional outcomes included a widely distributed book manuscript and a major research program at Royal Roads University based on the online process by which the book came about. 


\section{E-DIALOGUES RESEARCH PROGRAM}

Building upon the original objectives of NRTEE to "bring some of the best minds to bear on the problem" of sustainable development and recognizing the importance of interdisciplinary research based on my doctoral research, a research program was designed to answer the following three questions about online dialogue:

1. Can Internet communications technologies (ICTs), and e-dialogues in particular, be used for substantive dialogue?

2. Can ICTs be used to increase literacy, particularly when it concerns sustainable development?

3. Can e-dialogues be used to influence the public policy community to recognize critical public tensions around key issues?

This paper, which explores the evolution of a nascent research methodology that began with the creation of a multistakeholder process in 1988, addresses the first question only. All three questions can be addressed using e-dialogues. E-dialogues are deliberately designed, synchronous, online spaces that help bring together leading-edge researchers and practitioners and their diverse perspectives to address critical public policy issues. These real-time conversations are designed to increase key literacies, namely ecological literacy and civic literacy concerning public policy issues.

To date, five expert e-dialogues have been used to address climate change, social capital and sustainable community development, sustainable communities, the recruitment of the "scientist of the future," and the management of used nuclear fuel in Canada. As well, three students at Royal Roads University have used e-dialogues as a method for collecting data on topics as diverse as spirituality and sustainable development, cosmology and sustainable development, and the costs and benefits of "green" buildings.

This paper will now focus on the student-led edialogues and what they revealed regarding the potential of e-dialogue as a new e-research methodology for graduate students worldwide. For example, student-led e-dialogues can take advantage of economies of scale and the capacity of the Internet to bring diverse expertise together independent of place to build interdisciplinary espaces.

The student-led e-dialogues built upon networks built by the students as well as the research and professional networks provided by their thesis supervisors. Combining younger and older networks improved the diversity of experts willing to participate in these online discussions. The first student-led e-dialogue was moderated by the thesis supervisor independent of the student, whereas the other two involved different degrees of comoderation by the students. This methodology was particularly well-suited to the areas of research, given the breadth of the first two topics and their cross-disciplinary reach. The third e-dialogue, on green buildings, required bringing together a diversity of practitioners involved in the field and involved architects, government officials, and representatives from nongovernment organizations. For a critical evaluation of the latter, please refer to the Gallon Environment Letter at www.cialgroup.com/gallonletter.html.

The spirituality and sustainable development edialogue was the pilot student-led dialogue. It built upon the methods used in previous expert dialogues and involved no new variables. Although the student did not participate directly in the online dialogue, she shaped the discourse by developing the agenda and strategic questions for the three-day dialogue using traditional survey/interview research protocols. This was critical to her research. A purposive sample (Patten 1990), the dialogue brought together 20 experts. Eight were male and 12 were female. Representatives from academia, government, the private sector and civil society numbered eight, five, three, and four, respectively. Panelists were drawn from a diversity of disciplines, including theology, planning, social capital, sustainable community development, philosophy, ecology, and environmental ethics. They represented a variety of perspectives, including the views of academics, governments, and nongovernment organizations. No audience discussion was included, but 125 nonparticipants followed the dialogue. Several months after the dialogue's conclusion, interviews with seven of the 19 participants provided further insight into how to mobilize the connections between spirituality and sustainability. The interviews contributed to the 
student's triangulation of data.

The second student-initiated e-dialogue on cosmology and environmental education also involved the student shaping the agenda and strategic research questions. Unlike the first edialogue, however, the student actively participated and co-moderated the dialogue. There were 11 experts involved in this dialogue. The expert panel in this case did not remain the same for the three days. Panelists from the first and second day were combined on the third day. This change introduced another variable. Five participants were academics, one from government, and five from civil society. Seven of the panelists were male and four were female. Disciplines represented included theology, education, environmental communications, religion, philosophy, and psychotherapy. In addition, this edialogue involved two scholars from outside Canada. This dialogue was followed online by 182 nonparticipants.

The third student-led e-dialogue on the costs and benefits of green buildings differed from the previous two by leading a discussion on only one day instead of three. Of the seven expert panelists, six were male and one was female. One was an academic, one from government, three from the private sector, and two from civil society. The dialogue was followed by 81 nonparticipants. One interesting finding to emerge from this e-dialogue, which was primarily dominated by practitioners, was a distinction between green buildings and sustainable buildings. Panelists concluded that the former was a building that had been modified by additions and therefore involved a cost premium. On the other hand, the latter was a building that was built from an integrated design from the very beginning and thus appeared to have no cost premium. The student is now verifying this preliminary finding using a snowball sampling technique.

Table 1 shows the degree of engagement and level of interactivity by expert panelists involved in the online dialogues. The degree of engagement was measured using the number of postings, and the level of interaction was determined using the pace at which the postings appeared. Given the complexity of the subject matter, the online medium did not appear to inhibit interaction or trust in terms of engaging in an immediate and public space. The daily change in experts in the second e-dialogue did not appear to affect either the continuity of the dialogue or the building of trust between the experts. This was a surprise. The subject of the dialogue may dictate comfort levels with respect to trust and a willingness to engage in open dialogue, but additional research is required to verify this observation.

In these e-dialogues, the size of the panel affected the degree of interactivity (Table 1). Given the novelty of the medium and some of the skills it requires, such as typing and fast reading, group size online may be even more critical to meaningful dialogue and interaction than it is in face-to-face meetings in which 10 to 15 people is the optimal number for substantive dialogue. Online dialogue was improved when groups were smaller. Some software issues remain to be resolved, such as slight delays between responses. However, these delays also allowed for more reflection between postings and for time to reference additional print, presentation, or other materials as part of the conversation.

Dialogue on the Internet appears to improve the content and quality of the material by providing a "living archive." This term was suggested to me by government public policy experts who believe that archived conversations could be invaluable as an active reference tool. The first two student-led edialogues resulted in three other substantive outcomes: they successfully used a novel research methodology; they led to the successful defense of the students' broad, interdisciplinary theses; and they allowed for the dissemination of their research atwww.e-dialogues.ca for other young scholars to access. This last result allows other students to build upon these living archives as the intellectual capital is being created. The third student expected to complete his thesis in April 2005.

Each e-dialogue was supported by a Web site that offered a description of the issue under discussion, as well as a list of resources with links to additional Web sites and blogs for further information. Other research into online public forums has shown the benefits of being able to append reference material directly into the conversation. This increases access by the wider public to research information relevant to the topic under discussion.

The Web sites developed for each public policy issue in the field of sustainable development appeared to attract interest from a diversity of geographically dispersed people. In the five days preceding and following each online dialogue, 260 
unique visitors viewed the spirituality and sustainable development Web site, 619 visited the cosmology and environmental education Web site, and 243 unique visitors went to the green buildings Web site. The most recent e-dialogue on the management of nuclear waste management in Canada reached more than 2700 Canadians in a four-month period. Methods are now being developed to test the ability of these dialogues to increase literacy and to building upon a definition of sustainable development literacy (Dale and Newman, in press). A pre- and post-dialogue survey is underway.

It remains to be seen whether new research networks of collaboration will form between the expert panelists and the audiences participating in each dialogue, and whether new e-research networks will be stimulated because of the student-led initiatives. More importantly, e-dialogues may serve as an important tool for augmenting access by the wider public to intellectual capital within the academy. In the long run, this could enhance the literacy of the general public with respect to critical public policy issues.

\section{CONCLUSIONS}

The Internet appears to allow for both breadth and depth of dialogue and to permit both linear and lateral thinking. The students found that the Internet's linearity imposed a critical discipline for organizing broad interdisciplinary topics through the creation of their Web sites. Although sustainable development issues are amorphous and interconnected, they require some delimiting boundaries to facilitate meaningful dialogue. Some preliminary evidence also indicates that the Internet facilitates lateral as well linear thinking $(\mathrm{H}$. Regier, personal communication). This evidence is based on observation. Software development to test this is under way.

Many other potential applications of the software and the process also need to be tested. For example, it would be useful to examine the utility of edialogue methods as a means of sampling e-focus groups, as an advisory tool to governments, and as a means for conducting Delphi surveys.

E-dialogues are proving to be effective in online curriculum development and have now been incorporated into three online courses at Royal
Roads University. They mimic the traditional practice of bringing expert speakers into the classroom, and they are very popular with students. This is because they broaden student access to some of the leading-edge researchers in their fields of interest, both nationally and internationally. Time differences make the participation of international researchers more problematic. The students enjoy the dynamic interaction in real time with experts in a manner that complements their asynchronous classroom time. Finally, students can experiment with simultaneously dialoguing and searching the Internet for references to introduce into their discussions. Interestingly, moderation of classroom e-dialogues is more difficult than moderating expert panels because younger people have a computer literacy and ability to read on the screen that is unparalleled in older generations.

On the basis of my research project and its subsequent curriculum applications, I believe that e-dialogue has a powerful ability to gather together a variety of perspectives about a defined area of interest in a permissive, nonthreatening environment (Burton 2000, Mann and Stewart 2000). In this way, e-dialogue is an invaluable pedagogical tool. More importantly, e-dialogue also offers a novel way to minimize the constraints of time and space and to facilitate the triangulation of data (Glesne 1999) for student research.

Young scholars push the envelope, encouraging Internet uses as varied as online, e-mail coresearcher discussion groups, student-led edialogues, and a potential new e-research dialogue methodology. The latter novel methodology achieves many critical research objectives. First, it provides for greater interdisciplinary research. This is particularly important to sustainable development research in which dialogue is critical to bridge the differences between researchers working in different paradigms (Maxwell 2004) and the differences between the natural and social sciences, in particular. Second, the new methodology allows for enhanced connectivity between scholars, nationally and internationally, by bringing together in cyberspace scholars who would not normally meet or collaborate. Third, the methodology is a dynamic new way of student research dissemination that increases access to intellectual capital through the living archive. Fourth, it provides direct application of research work by bringing together experts and practitioners around common critical issues. Fifth, it may create a new kind of civic 
literacy.

Sixth, the new methodology may contribute directly to the eventual formation of two levels of research networks, new e-research collaborations of scholars and new communities of practice involving researchers and practitioners. Seventh, the methodology provides an important means to reengage graduate students in the online development of intellectual capital in their respective areas of interest. Graduate cohorts can use the methodology to stay in touch with one another, to continue to bridge theory and practice, and to keep their research skills finely honed. This will help to advance critical public policy development for those following careers in the public sector.

Finally, this e-research methodology may contribute to other emergent ways to encourage research discourse, "the discourse, not a closure but a trace in an endless passage that can only aspire to a temporary arrest, to a self-conscious drawing of a limit across the diverse possibilities of the world" (Chambers 1990).

Responses to this article can be read online at:

http://www.ecologyandsociety.org/vollo/iss1/art37/responses/

\section{Acknowledgments:}

I wish to acknowledge the support for this research project provided by Steve Grundy and Mary Bernard, as well as the research assistance of Isabel Cordua-von Specht and the invaluable work of Darren Oxner. I also wish to thank the Social Sciences and Humanities Research Council of Canada for its support.

\section{LITERATURE CITED}

Burton, D. 2000. Research training for social scientists: a handbook for postgraduate researchers. Sage Publications, London, UK.

Chambers, I. 1990. Border dialogues: journeys in postmodernity. Routledge, Oxford, UK.

Dale, A., and L. Newman. In press. Sustainable development, education and literacy. Journal of Sustainability in Higher Education.

Dale,A. 2001. At the edge: sustainable development in the $21^{\text {st }}$ century. University of British Columbia Press, Vancouver, British Columbia, Canada.

Glesne, C. 1999. Becoming qualitative researchers: an introduction. Routledge, Oxford, UK.

May, P.J., R.J. Burby, N.J. Ericksen, J.W. Handmer, J.E. Dixon, S. Michaels, and D.I. Smith. 1974. Environmental management and governance: intergovernmental approaches to hazards and sustainability. Routledge, Oxford, UK.

Mann, C., and F. Stewart. 2000. Internet communication and qualitative research: $a$ handbook for researching online. Sage Publications, London, UK.

Maxwell, J.A. 2004. Reemergent scientism, postmodernism, and dialogue across differences. Qualitative Inquiry 10:35-41.

Michael, D.N. 1993. Governing by learning: boundaries, myths and metaphors. Futures 25:81-89.

Morgan, G. 1986. Images of organization. Sage Publications, Thousand Oaks, California, USA.

Norgaard, R. 1994. Development betrayed: the end of progress and a co-evolutionary revisioning of the future. Routledge, New York, New York, USA.

Patten, M.Q. 1990. Qualitative evaluation and research methods. $2^{\text {nd }}$ edition. Sage Publications, Thousand Oaks, California, USA.

Peterson, G., G. De Leo, J. Hellmann, M. Janssen, A. Kinzig, J. Malcolm, K. O'Brien, S. Pope, D.S. Rothman, E. Shevliakova, and R. Tinch. 1997. Uncertainty, climate change, and adaptive management. Conservation Ecology 1:4 (Online) URL: http://www.consecol.org/voll/iss2/art4.

Roberts, N.C., and R.T. Bradley. 1991. Stakeholder collaboration and innovation: a study of public policy initiation at the state level. Journal of Applied Behavioral Science 27:209-227.

Shermer, M. 2004. Common sense: surprising new research shows that crowds are often smarter than 
individuals. Scientific American December 2004. (Online) URL:

http://www.sciam.com/article.cfm?articleID=00049F3E-91E1-119B-8EA483414B7FFE9F.

Schon, D. 1983. The reflexive practitioner. Basic Books, New York, New York, USA.

Rowan, J. 1981a. On making sense. Pages 113-136 in

P. Reason and J. Rowan, editors. Human inquiry: a sourcebook of new paradigm research. John Wiley and Sons, New York, New York, USA.

Rowan, J. 1981b. A dialectical paradigm for research. Pages 19-36 in

P. Reason and J. Rowan, editors. Human inquiry: a sourcebook of new paradigm research. John Wiley and Sons, New York, New York, USA.

Wellman, B., J. Salaff, D. Dimitrova, L. Garton, M. Gulia, and C. Haythornthwaithe 1996. Computer networks as social networks: collaborative work, telework, and virtual community. Annual Review of Sociology 22:213-238. 